\title{
Stationsäquivalente Behandlung
}

\author{
Rechtliche und organisatorische Rahmenbedingungen
}

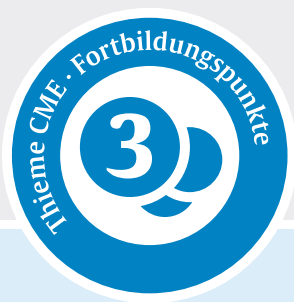

Gerhard Längle ${ }^{1,2,4}$, Svenja Raschmann², Martin Holzke²,3

1 Klinik für Psychiatrie und Psychosomatik Reutlingen PP.rt

2 ZfP Südwürttemberg

3 Klinik für Psychiatrie und Psychotherapie I der Universität Ulm

4 Universitätsklinik für Psychiatrie und Psychotherapie Tübingen

\section{ZUSAMMENFASSUNG}

In Deutschland herrscht nach wie vor eine strikte Sektorisierung der Versorgungslandschaft. Aufsuchende Akutbehandlung war bislang nur in Modellvorhaben realisiert und nicht bundesweit umsetzbar. Mit dem Inkrafttreten des Gesetzes zur Weiterentwicklung der Versorgung und Vergütung für psychiatrische und psychosomatische Leistungen (PsychVVG) im Jahr 2017, wurde diese Versorgungslücke mit der Stationsäquivalenten Behandlung (StäB) als neuartige Form aufsu- chender Akutbehandlung gefüllt. In diesem Beitrag werden die für StäB relevanten rechtlichen Grundlagen und die in der Selbstverwaltung getroffenen Vereinbarungen gebündelt. Bisher bekannte Modelle der Finanzierung werden vorgestellt und der Umgang mit StäB aus Sicht der Krankenhausplanung wird dargelegt. Auch in der Kinder- und Jugendpsychiatrie ergänzt die stationsäquivalente Behandlung die Landschaft psychiatrischer Versorgungsangebote, weshalb deren besondere Anforderungen an die Behandlung im Zuge der StäB gesondert aufgegriffen werden. Aus aktuellem Anlass nimmt der Beitrag Bezug auf die Erfahrungen mit StäB in Zeiten der SARS-CoV-2 Pandemie. StäB wurde während sozialer Kontaktsperren und Quarantäne- und Hygienemaßnahmen an den verschiedenen Zentren unterschiedlich ausgestaltet. Allen gemeinsam waren die erforderlichen Anpassungen an die Hygiene- und Abstandsregelungen, welche nochmals komprimiert dargestellt werden.

\section{Einführung}

Im Kontext der deutschen Sozialgesetzgebung war aufgrund der klaren Grenzen zwischen dem ambulanten und dem stationären Sektor eine flächendeckende und bundesweite Umsetzung aufsuchender Akutbehandlung nicht vorgesehen. So fasste auch der Sachverständigenrat zur Entwicklung im Gesundheitswesen in seinem Gutachten zusammen, dass zwischen ambulanter und stationärer Versorgung nach wie vor große Hürden bestehen [1]. Lediglich über die Realisierung in Form von Modellvorhaben wie z. B. Modelle der integrierten Versorgung nach $\S 140$ SGB V oder nach §64 SGB V konnten einzelne, regionale Modelle erprobt werden. Alternativ war eine Realisierung, verbunden mit dem Sammeln von Erfahrungen, nur auf Basis von Forschungsprojekten, finanziert aus Forschungsmitteln, möglich. Einigen wenigen Bundesländern (Bayern, Sachsen) war es aufgrund der landesspezifischen Vereinbarungen zur Psychiatrischen Institutsambulanz (PIA) in gewisser Weise möglich, eine ambulante aufsuchende Behandlung zu intensivieren, nicht jedoch in demselben Umfang, den die stationsäquivalente Behandlung (StäB) nun ermöglicht.

Die vielfältigen Erfahrungen hierzu trugen möglicherweise entscheidend dazu bei, dass die aufsuchende Akutbehandlung in das Gesetz zur Weiterentwicklung der Versorgung und Vergütung für psychiatrische und psychosomatische Leistungen (PsychVVG) gemäß den Drucksachen
$18 / 9528$ und 18/10289 aufgenommen wurde [2, 3]. Nachdem dieses zum Jahresbeginn 2017 in Kraft getreten ist, gibt es in Deutschland für akut psychisch Erkrankte bei vorhandener stationärer Behandlungsbedürftigkeit nun auch die Möglichkeit, zu Hause stationsäquivalent behandelt zu werden.

StäB bewegt sich hierbei im Spannungsfeld der Zuständigkeiten zwischen den Partnern der Selbstverwaltung im Gesundheitswesen, Bund und Ländern und den engen Vorgaben der Sozialgesetzbücher. Mit der neuen Form aufsuchender Akutbehandlung können bestehende Behandlungsangebote (ambulant, teilstationär, stationär) komplettiert werden, sodass in einer akuten Krankheitsphase eine weitere Alternative zur Verfügung steht. Auch dass diese Behandlungsform ungeachtet der Versorgungsregion, der durchführenden Institution oder der Zugehörigkeit zu einer spezifischen Krankenkasse prinzipiell allen psychisch kranken Patienten zur Verfügung steht, unterstützt den sozialpsychiatrischen Ansatz, das direkte Lebensumfeld des Patienten sowie dessen soziale Interaktionen in die Behandlung zu integrieren [4]. Mit der stationsäquivalenten Behandlung, als intensive und wohnortnahe Versorgungsform wurde ein wesentlicher Fortschritt in der Umsetzung der Grundsätze der Psychiatrie-Enquete von 1975 erreicht ( $\bullet$ Abb. 1). Es ist somit ein wichtiger Schritt zur Verbesserung der wohnortnahen Patientenversorgung im Akutbereich gegeben, wobei die Hürden in der Flexi- 


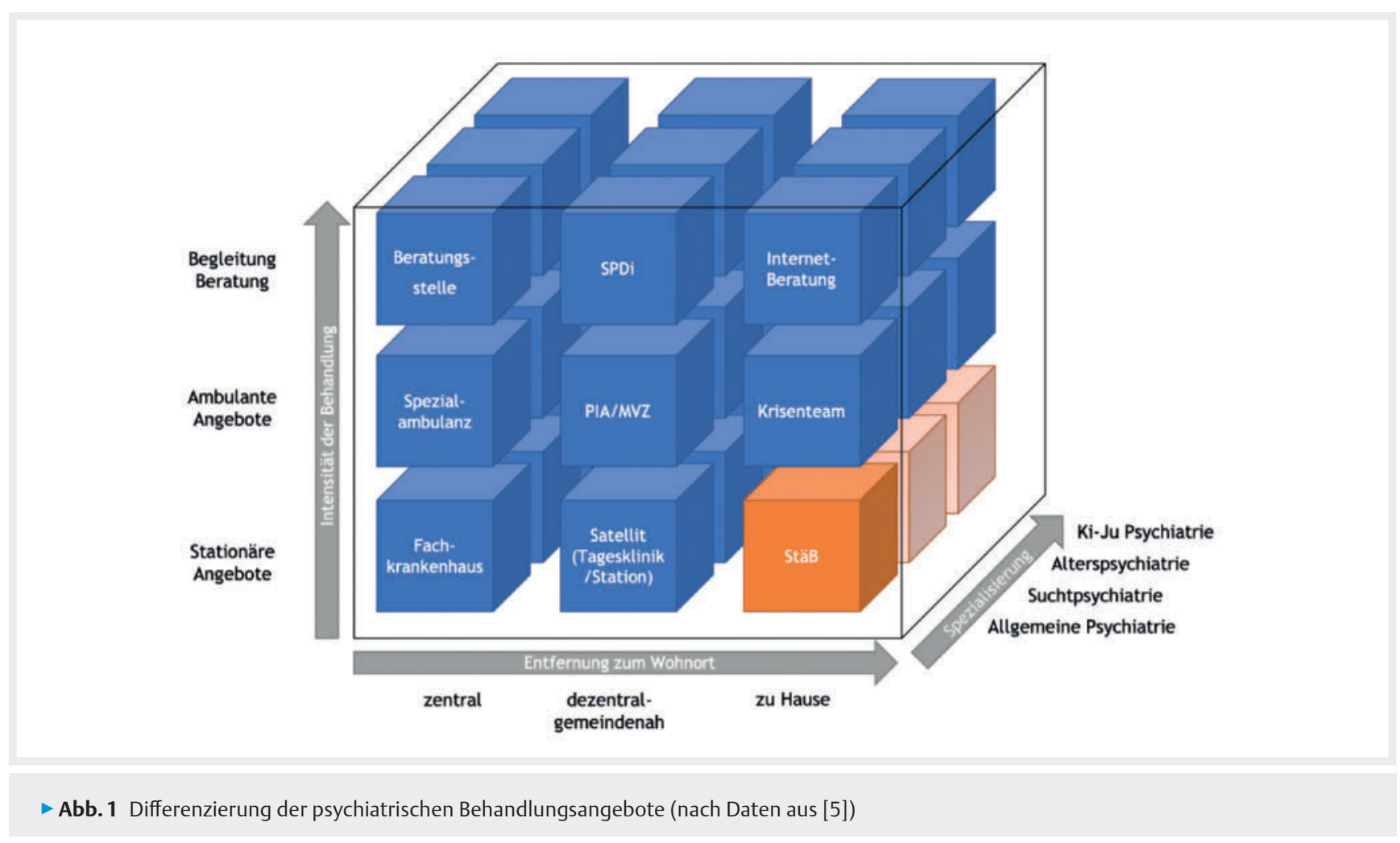

bilisierung der strikten Sektorisierung dennoch in Teilen weiter bestehen bleiben.

\section{Gesetzliche Grundlagen und Verordnungen}

Die zur stationsäquivalenten Behandlung bestehenden gesetzlichen Grundlagen sind größtenteils im PsychVVG niedergelegt und finden sich auch im Sozialgesetzbuch in der Neufassung des $\S 39$ Abs. 1 SGB V sowie in $\S 115 \mathrm{~d}$ SGB V wieder. Einen umfassenden Überblick dazu bietet auch das Handbuch zur stationsäquivalenten Behandlung [6]. Grundsätzliches zur neuen Behandlungsform wird in $\S 39$ Abs.1 SGB V geregelt:

„Die Krankenhausbehandlung wird stationär, stationsäquivalent, teilstationär, vor- und nachstationär sowie ambulant erbracht; ...Versicherte haben Anspruch auf vollstationäre oder stationsäquivalente Behandlung durch ein nach $\S 108$ zugelassenes Krankenhaus, wenn die Aufnahme oder die Behandlung im häuslichen Umfeld nach Prüfung durch das Krankenhaus erforderlich ist, weil das Behandlungsziel nicht durch teilstationäre, vor- und nachstationäre oder ambulante Behandlung einschließlich häuslicher Krankenpflege erreicht werden kann. .... Die stationsäquivalente Behandlung umfasst eine psychiatrische Behandlung im häuslichen Umfeld durch mobile ärztlich geleitete multiprofessionelle Behandlungsteams. Sie entspricht hinsichtlich der Inhalte sowie der Flexibilität und Komplexität der Behandlung einer vollstationären Behandlung." [7]
Die Behandlung im direkten Lebensumfeld des Patienten mit all seinen Vorteilen, schließe neben dem privaten häuslichen Umfeld auch eine Behandlung im Alten- oder Pflegeheim als unmittelbaren Lebensmittelpunkt von Patienten mit ein [8]. Als Voraussetzung gelte die fortwährend bestehende stationäre Behandlungsbedürftigkeit [2, 8]. Eine Behandlung soll demnach während der gesamten notwendigen Behandlungsdauer stationsäquivalent erbracht werden können und bei Erfüllung der leistungsrechtlichen Voraussetzungen obliege es der Entscheidung des Krankenhauses, ob die Behandlung vollstationär oder stationsäquivalent durchgeführt wird [8].

Die Zuständigkeit bei neu auftretenden Begleiterkrankungen, welche während einer stationsäquivalenten Behandlung aufkommen, war von Beginn an beim psychiatrischen Krankenhaus angesiedelt. Eine analoge Anwendung der Grundsätze zur Mitbehandlung interkurrenter Erkrankungen wie bei vollstationärer Behandlung gestaltete sich für StäB zum Teil aber sehr schwierig. Bei der Frage der Übernahme von Pflegeleistungen im Heim hat sich das Bundesministerium für Gesundheit klar positioniert. Die Verantwortung für die Pflege bleibe weiterhin beim Heim und sei unberührt von der stationsäquivalenten Behandlung [9]. Die anfänglich bestehende Unsicherheit bezüglich der Übernahme häuslicher Krankenpflege durch ambulante Dienstleister wurde inzwischen ebenso mit der Änderung der häuslichen Krankenpflegerichtlinie ausgeräumt. Diese legt fest, dass auch die häusliche Krankenpflege Teil der StäB-Leistung ist und somit aus dem StäB-Team heraus erbracht werden muss [10]. 
Weitere Regelungen sind in §115 d SGB V getroffen. Dort ist festgeschrieben, dass neben psychiatrischen Krankenhäusern mit regionaler Versorgungsverpflichtung auch Allgemeinkrankenhäuser mit entsprechenden psychiatrischen Abteilungen eine stationsäquivalente Behandlung erbringen können. Der Krankenhausträger stellt demnach notwendiges Personal zur Gewährleistung der Behandlungskontinuität sowie zur Sicherstellung einer Notfallversorgung zur Verfügung. Ebenso wird geregelt, dass ambulante psychiatrische Leistungserbringer durch das Krankenhaus mit der Durchführung von Teilen der StäB beauftragt werden können [7]. Weiter ist hier niedergelegt, dass der Spitzenverband der Krankenkassen und der Verband der privaten Krankenversicherung sowie die Deutsche Krankenhausgesellschaft (DKG) weitere Anforderungen in einer Rahmenvereinbarung definieren.

\section{Bundesrahmenvereinbarung}

Die Rahmenvereinbarung zur stationsäquivalenten psychiatrischen Behandlung nach §115 d Abs. 2 SGB V ist in einer Veröffentlichung der DGK aus dem Jahr 2017 nachzulesen [8]. Die Vereinbarung klärt neben dem Geltungsbereich unter anderem Qualitätsanforderungen bezüglich der Leistungserbringung. So wird beispielsweise festgehalten, dass die Behandlung im häuslichen Umfeld durch mobile, fachärztlich geleitete multiprofessionelle Behandlungsteams erfolgen soll [8]. Ebenso wird festgehalten, dass die Behandlung nach Intensität, Flexibilität und Komplexität einer vollstationären Behandlung entspricht [8]. Auch weitere, speziell für die stationsäquivalente Behandlung geltende Vereinbarungen, werden hier definiert. Zum Beispiel eine Behandlungsfrequenz und -intensität von zumindest einem täglichen persönlichen Kontakt durch ein Mitglied des Behandlungsteams sowie die Prüfung des häuslichen Umfeldes auf Eignung hinsichtlich räumlicher Möglichkeiten und der Vermeidung einer drohenden Kindeswohlgefährdung. Die weiteren Regelungen sind im Detail in der Rahmenvereinbarung nachzulesen.

Die Möglichkeit der Einbindung außerklinischer Leistungserbringer in StäB ist ebenfalls in der Rahmenvereinbarung festgeschrieben. Hier wird der Verbleib der Gesamtverantwortung beim aufnehmenden Krankenhaus sowie eine Obergrenze zur Auslagerung von Behandlungstätigkeiten, welche vorgibt, dass maximal die Hälfte der Behandlungstätigkeiten delegiert werden dürfen, definiert [8]. Vor allem in der Gemeinde- und Sozialpsychiatrie wurde dieses Thema vielfach diskutiert, weshalb auch die DGPPN in Ihrer Stellungnahme zur Umsetzung darauf Bezug nahm [11]. Mit der sich langsam einstellenden Routine in der aufsuchenden Akutbehandlung, beginnen nun erste Krankenhäuser damit, sich dem Thema der intensiven Einbindung krankenhausexterner Leistungsanbieter verstärkt zu widmen. Erste positive Erfahrungen konnten mit der Integration von niedergelassenen Psychotherapeuten gesammelt werden. Auch andere ambulante Leistungserbringer wie betreute Wohneinrichtungen oder der sozialpsychiatrische Dienst werden inzwischen teilweise eingebunden. Problematisch zeigt sich in der Praxis aber vor allem die Einbindung von nicht in einer eigenen Praxis arbeitenden Leistungserbringern, bei welchen der Auffassung einzelner Fachanwälte zufolge dann die Vorgaben aus dem Arbeitnehmerüberlassungsgesetz zum Tragen kommen. Die Einschätzungen einzelner Juristen differieren hierbei aber stark voneinander. Eine eindeutige rechtliche Positionierung zum Umfang und der rechtlichen Konstruktionen und Voraussetzungen, unter welchen eine Einbindung von Mitarbeitenden externer Institutionen in das StäB-Behandlungsteam erfolgen kann, existiert nicht. Eine konkrete Klärung wird sich aber in den nächsten Jahren sicherlich ergeben, da sowohl Krankenhäuser als auch ambulante Leistungsanbieter immer stärker die Vorteile enger Zusammenarbeit erkennen und sich im Hinblick auf eine Verbesserung der Patientenversorgung diese auch verstärkt wünschen. Weitere wichtige Punkte, welche in der Rahmenvereinbarung niedergelegt sind, betreffen die Anforderungen an die Dokumentation, sowie auch die differenzierte Leistungsbeschreibung innerhalb des OPS-Katalogs.

\section{Dokumentation}

Im Allgemeinen sind die gestellten Anforderungen an die Dokumentation vergleichbar mit denen im stationären Setting. Im Detail können diese Regularien in der Rahmenvereinbarung nachgelesen werden. Als einzelne Besonderheiten möchten wir an dieser Stelle beispielhaft die Einholung der Zustimmung der volljährigen im Haushalt lebenden Personen sowie die Prüfung des häuslichen Umfeldes auf Eignung nennen.

Nachdem in den ersten beiden Jahren zahlreiche Prüfungen durch den medizinischen Dienst der Krankenkassen (MDK) erfolgten, zeigten sich vor allem gehäuft Rückfragen bezüglich der formalen Rahmenbedingungen und genannter beispielhafter Anforderungen an die Dokumentation. In den Zentren für Psychiatrie Südwürttemberg und der Klinik für Psychiatrie und Psychosomatik Reutlingen (PP.rt) wurde aus diesen Erfahrungen heraus für die Vorgaben der Dokumentation eine Checkliste erstellt. Es erwies sich als praktikabel, die Inhalte individuell für jeden StäB-Patienten niederzulegen.

\section{OPS}

Auch die differenzierten Beschreibungen der Leistungsinhalte der einzelnen Berufsgruppen stimmen im Wesentlichen mit den Leistungsinhalten der stationären Behandlung überein. Sie wurden im Rahmen des DIMDI-Prozesses im Jahr 2017 ausgearbeitet (OPS 9-701). Auch für die stationsäquivalente psychiatrische Behandlung von Kindern und Jugendlichen steht analog eine differenzierte Leistungsbeschreibung zur Verfügung (OPS 9-801). Die Therapiezeiten am Patienten werden hierbei differenziert nach Berufsgruppe (Ärzte, Psychologen, Spezialtherapeuten, Pflegefachpersonen) sowie der jeweiligen Zeiteinheit 
pro Tag in Minuten. Dieser Prozess unterliegt immer wieder Veränderungen, sodass im Katalog 2020 auch ergänzend die Möglichkeit der Kodierung von kriseninterventioneller Behandlung im StäB-Kontext ergänzt wurde [12].

\section{Richtlinie zur Personalausstattung in Psychiatrie und Psychosomatik (PPP-RL)}

Auch die im PsychVVG geforderte und am 31.12.2019 veröffentlichte PPP-RL [13] bezieht sich auf StäB. Für Patienten in stationsäquivalenter Behandlung ist die Einstufung in die Kategorie A9, S9, G9 oder KJ9 vorzunehmen. In StäB sind prinzipiell alle Diagnose- sowie Altersgruppen vorgesehen, exakte Minutenwerte sind jedoch noch nicht hinterlegt. Diese können erst auf Basis empirischer Daten festgelegt werden. Auch das StäB-Team selbst wird daher vom Personalnachweis nicht erfasst. Langfristig wird diesbezüglich jedoch angestrebt, die krankenhausindividuellen Vergütungen durch auf Bundesebene kalkulierte Entgelte abzulösen, welche sich auf der Grundlage von Kostenund Leistungsdaten psychiatrischer Krankenhäuser ergeben. Hierfür wurde ein Zeitrahmen von 5 Jahren fixiert.

\section{Stationsäquivalente Behandlung in der Kinder- und Jugendpsychiatrie}

Psychische Auffälligkeiten, welche einer Behandlung, zumindest jedoch einer weiteren Abklärung bedürfen, sind auch bei Kindern und Jugendlichen in Deutschland keine Ausnahme und besitzen gemäß Ergebnissen der in Deutschland durchgeführten KIGGS-Studie eine Prävalenz von rund $20 \%$ [14]. Der Zugang zu den Versorgungsangeboten im ambulanten sowie stationären Sektor ist regional jedoch sehr unterschiedlich ausgeprägt [15]. Allen gemeinsam ist in diesem Bereich der psychiatrischen Versorgung die lange Zeit vorherrschende strikte Trennung der beiden Sektoren gewesen. Eine aufsuchende Akutbehandlung war somit auch in der Regelversorgung für psychisch erkrankte Kinder- und Jugendliche weder vorgesehen noch finanziert.

Dies war umso bedauerlicher, weil gerade in der Behandlung von Störungen des Kindes- und Jugendalters der Einbezug des sozialen Umfelds einen enorm hohen Stellenwert einnimmt. Neben relevanten Beeinträchtigungen für die betroffenen Kinder und Jugendlichen werden auch Störungen im gesamten sozialen System der Betroffenen, beispielsweise in der Familie oder der Peergroup angenommen [16]. Dies wird in einem Bericht des Vereins „Aktion psychisch Kranke e. V.“ zur Versorgung psychisch kranker Kinder- und Jugendliche in der Form beschrieben, dass Kinder und Jugendliche nie ohne ihr betreuendes Umfeld oder das familiäre System behandelt werden sollten [17]. Daher wurden die erwähnten Bestrebungen in der Erwachsenenpsychiatrie zur Intensivierung von Behandlungen im direkten häuslichen Umfeld ausgeweitet. Auch für Kinder und Jugendliche wurden erste Formen von intensiver „Zuhause Behandlung “ in Modellprojekten erprobt und evaluiert [18]. Für Kinder und Jugendliche wird der Zugang ins Hil- fesystem erleichtert, da diese weiter inmitten des eigenen Familiensystems verbleiben können und nicht dem Risiko einer Ausgrenzung aus der Schule oder der sozialen Peergroup ausgesetzt sind. Auch können in diesem Setting weitgehend die alltäglichen Strukturen im Tagesablauf aufrechterhalten werden [16]. Diese Überlegungen wurden durch unterschiedliche deutsche und internationale Studien gestützt, welche vergleichbare Behandlungsergebnisse bei stationärer oder Hometreatment-Behandlung finden konnten [19]. Andere Untersuchungen wiesen auf eine Überlegenheit von Hometreatment in Bezug auf die 1-Jahres-Stabilität hin [20].

Mit dem Inkrafttreten des PsychVVG wurde Fachkrankenhäusern mit regionaler Versorgungsverpflichtung auch im Bereich der Kinder- und jugendpsychiatrischen Versorgung in Form der StäB die lang ersehnte Möglichkeit einer intensiven bedarfsorientierten Behandlung zuhause eingeräumt. Patienten kann eine Behandlung angeboten werden, welche auf der einen Seite eine höhere Intensität möglich macht als ein ambulantes Angebot, die Belastung durch ein oft sehr intensives tagesklinisches Setting aber nicht erreicht sowie die befürchtete Stigmatisierung durch einen stationären Aufenthalt vermeidet. Die in der Behandlung von Kindern und Jugendlichen zu beachtenden Notwendigkeiten wie der Einbezug eines ganzen Familiensystems oder eine Integration der Schule in die Behandlung können in StäB ebenso berücksichtigt werden. Vertreter der KJPP und die Betroffenenverbände sind sich, trotz der noch zögernden Umsetzung gerade in diesem Bereich, einig, dass das Behandlungsangebot durch StäB verbessert wird. Auch die nachgewiesene hohe Akzeptanz in den Familien unterstützt dies [15].

\section{Finanzierung}

Die finanzielle Vergütung der in der stationsäquivalenten Behandlung erbrachten Leistungen ist grundsätzlich im Krankenhausgesetz geregelt [21]. Details sind darin nicht ausgearbeitet, sodass die anfängliche Finanzierung individuell in den jeweiligen Pflegesatzverhandlungen verhandelt wurde. Die Ansichten bezüglich der tatsächlichen Kosten einer aufsuchenden Akutbehandlung in Form von StäB differierten hierbei stark. Da die Spannweite der Vorstellungen von einem deutlichen Mehrbetrag zum stationären Pflegesatz (zum damaligen Zeitpunkt knapp 350 Euro) bis hin zu einem deutlich geringeren Mindestbetrag reichte, wurde vorläufig ein Erstattungsbetrag festgelegt. Dieser entspricht pro Fall und Tag für unbewertete PEPPs insgesamt 200 Euro [22]. So wurde sichergestellt, dass auch ohne Abschluss einer Vergütungsvereinbarung zumindest eine Abschlagszahlung möglich ist.

In der PP.rt Reutlingen wurden im Mai 2018 bundesweit die ersten Pflegesätze für StäB verhandelt. Nachdem verschiedene Finanzierungsmodelle durchgespielt wurden, einigte man sich auf eine zumindest teilweise leistungso- 
rientierte Vergütungsform zusätzlich zu einer pauschalisierten Vergütung. Die Tagespauschale setzt sich hierbei aus 2 Bestandteilen zusammen: Pauschale zur Abdeckung von Personal- und Sachkosten und Pauschale für sonstige patientenferne Tätigkeiten (z. B. Telefonate, die Erstellung von Stellungnahmen, sozialadministrative Tätigkeiten). Der leistungsorientierte Teil der Vergütung enthält neben einer berufsgruppenspezifisch berechneten Fahrtzeitpauschale pro Patientenkontakt von 40 Minuten auch die tatsächlich am Patienten verbrachte Zeit. Diese wird anhand der dokumentierten OPS je Berufsgruppe und Tag abgerechnet. Dieses Modell der Finanzierung wurde flächendeckend in Baden-Württemberg übernommen, teilweise wurden kleine Modifikationen der jeweiligen Summen vorgenommen.

Neben dem baden-württembergischen Modell ist ein weiteres Finanzierungsmodell bekannt, welches sich in Nordrhein-Westfalen, Brandenburg, Berlin und Hessen durchsetzte. Hier findet eine pauschalisierte Vergütung statt, welche sich an festen Tagessätzen, vergleichbar mit der Höhe der durchschnittlichen stationären Pflegesätze vor Ort, orientiert.

\section{Krankenhausplanerischer Umgang mit StäB}

Das PsychVVG enthält zur Krankenhausplanung keine verbindlichen Regelungen, da diese Aufgabe der jeweiligen Bundesländer ist. Dementsprechend sind die Regelungen zur Krankenhausplanung bundesweit vielfältig. Ein einheitlicher Umgang mit der Planung von Krankenhauskapazitäten für die StäB ist nicht absehbar. Auf bundespolitischer Ebene relativ klar formuliert wurde in der Entstehung des Gesetzes der Wunsch, vorhandene Bettenkapazitäten nicht auf die StäB-Behandlung anzurechnen oder einen Austausch zwischen Planbett und StäB-Behandlungsplatz vorzunehmen. Einer zunächst von Kassenseite geforderten direkten Reduktion von Klinikbetten entsprechend dem Aufbau der StäB-Behandlungskapazität wurde im Gesetzgebungsverfahren widersprochen. Ein entsprechender Passus wurde aus dem Referentenentwurf des Gesetzes wieder gestrichen. Die neue Behandlungsform sollte nicht von vorne herein durch eine solche Verrechnung ausgebremst werden. Nach unserer Kenntnis hat sich nur das Land Baden-Württemberg dem Thema krankenhausplanerisch gewidmet und eine Regelung vorgenommen. Diese tritt zum Juli 2020 in Kraft. Vorbereitet wurde sie in einer Arbeitsgruppe des Sozialministeriums Baden-Württemberg unter Beteiligung der Verbände der Krankenkassen und der baden-württembergischen Krankenhausgesellschaft unter Einbezug von klinischen Experten. Vor allem bundesweit agierende Krankenkassen wollten zunächst eine Verrechnung von Krankenhausbetten und StäB-Behandlungsplätzen durchsetzen. Angesichts des erklärten politischen Willens, in Baden-Württemberg stationsäquivalente Behandlung zu fördern, konnte jedoch eine sinnvolle Einigung getroffen werden: Krankenhäuser, die derzeit StäB noch nicht umsetzen, können per Antrag die zusätzliche Ausweisung von 5 StäB-Plätzen ohne Verrechnung mit der Behandlungskapazität im stationären Bereich beantragen. Bei einer durchschnittlichen Auslastung der Krankenhausbetten über $90 \%$ wird dem Antrag auch stattgegeben. Sofern die stationäre Auslastungskapazität geringer ist, kann über eine Verrechnung diskutiert werden. Häuser, die bereits eine gewisse Zeit StäB durchführen (in Baden-Württemberg sind dies 7 Kliniken), können die belegten Plätze (plus Entwicklungsoption) sowie ggf. eine Erweiterung von Plätzen aufgrund von Spezialisierung oder Regionalisierung beantragen. Konkret kann ein größeres Haus, das eine Satellitenabteilung betreibt, zusätzliche StäB-Plätze für die wohnortnahe Behandlung von den Satelliten aus beantragen sowie die Einrichtung von spezialisierten Teams, z. B. für Allgemeinpsychiatrie, Suchtpsychiatrie und Alterspsychiatrie. Mittel- bis längerfristig soll zudem die Entwicklung der StäB-Plätze in ein Verfahren ähnlich der stationären Behandlungskapazitäten überführt werden.

Die Zahl der Anträge und der Umfang der Genehmigung von StäB-Plätzen wird bis Ende des Jahres 2021 klarer einzuschätzen sein. Über die vorliegenden Anträge war zum Zeitpunkt der Drucklegung dieses Artikels noch nicht entschieden. Insgesamt kann das baden-württembergische Verfahren als modellhaft betrachtet werden. Um die Etablierung von StäB weiterhin zu fördern und zu sichern, sollte eine Verrechnung mit stationären Kapazitäten jedoch grundsätzlich vermieden werden.

\section{StäB in Corona-Zeiten}

Zu Beginn der Corona-Pandemie in Deutschland wurde unter den Kliniken, welche StäB seit längerer Zeit durchführen, intensiv diskutiert, ob diese Behandlung vorläufig eingestellt oder - im Gegenteil - intensiviert werden sollte. Vielerorts wurde die tagesklinische Behandlung stark reduziert oder phasenweise ganz aufgegeben. Ambulante Behandlung fand, in Absprache mit den Krankenkassen, verstärkt telefonisch oder per Videokonferenz statt. Bei StäB stand bezüglich der Gefährdungslage zunächst insbesondere die Gefährdung für die Mitarbeitenden, nicht so sehr durch die Mitarbeitenden im Fokus der Diskussion. Bei anfänglichem Mangel an Schutzausrüstung war unklar, welcher Gefährdungsgrad für die Mitarbeitenden angenommen werden kann und ob eventuell sogar alle StäB-Patienten als Verdachtsfälle zu betrachten seien.

An den 7 Standorten in Südwürttemberg, an denen StäB durchgeführt wird, bestand Einigkeit darüber, diese Behandlungsform auch in Corona-Zeiten fortzuführen. Als wesentlich erachtet wurde und wird das Abstandsgebot. Realisiert wird dies zum Teil durch Gespräche im Freien, durch gemeinsame Spaziergänge oder durch entsprechende Maßnahmen in der häuslichen Umgebung, jeweils unter 
Wahrung der Vertraulichkeit. Falls der Sicherheitsabstand von 1,5 m nicht eingehalten werden kann, werden von beiden Beteiligten MNS-Masken getragen. Jedes Fahrzeug war und ist mit der kompletten Covid-Schutzausrüstung ausgestattet, Händedesinfektion findet vor und nach jedem Kontakt statt, das Fahrzeug wird regelmäßig desinfiziert. Evtl. vor Ort genutztes Material wird verworfen oder desinfiziert. Die Behandlung von Verdachtsfällen oder manifest erkrankten Personen erfolgt in voller Schutzausrüstung (FFP2-Masken, Haube, Brille, Schutzkittel, Handschuhe).

Im Ergebnis wurde die Häufigkeit der aufsuchenden Behandlung am einzelnen Tag etwas reduziert, zumindest ein persönlicher Kontakt fand jedoch gemäß der Bundesrahmenvereinbarung jeden Tag statt. Videotelefonie und allgemeine Telefonkontakte wurden deutlich verstärkt genutzt. Von Seiten der Mitarbeitenden und der StäB-Patienten wurde dieses Vorgehen als positiv bewertet. Die Zahl der StäB-Behandlungen verlief je nach Standort etwas unterschiedlich, an vielen Standorten wurde die StäB-Behandlung vermehrt genutzt. Gerade die Sorge vor einer potenziellen Ansteckung im stationären Geschehen war für manche Patienten ein greifendes Argument für die stationsäquivalente Behandlung im eigenen häuslichen Umfeld. Nach unserer Erfahrung kann auch bei den geltenden Corona-Bestimmungen StäB weitgehend problemlos und ohne Einschränkung der Behandlungsqualität durchgeführt werden.

\section{Interessenkonflikt}

\section{Erklärung zu finanziellen Interessen}

Forschungsförderung erhalten: nein; Honorar/geldwerten Vorteil für Referententätigkeit erhalten: nein; Bezahlter Berater/interner Schulungsreferent/Gehaltsempfänger: nein; Patent/Geschäftsanteile/Aktien (Autor/Partner, Ehepartner, Kinder) an Firma (Nicht-Sponsor der Veranstaltung): nein; Patent/Geschäftsanteile/Aktien (Autor/Partner, Ehepartner, Kinder) an Firma (Sponsor der Veranstaltung): nein.

Erklärung zu nicht finanziellen Interessen

Die Autoren geben an, dass kein Interessenkonflikt besteht.

\section{Korrespondenzadresse}

Prof. Dr. med. Gerhard Längle

Klinik für Psychiatrie und Psychosomatik Reutlingen (pp.rt) Deutschland

Tel. 07121/9200110

gerhard.laengle@pprt.de

\section{Literatur}

[1] Sachverständigenrat zur Begutachtung der Entwicklung im Gesundheitswesen. Kurzfassung des Gutachtens 2018:
Bedarfsgerechte Steuerung der Gesundheitsversorgung. https://www.svr-gesundheit.de/fileadmin/user_upload/Gutachten/2018/SVR-Gutachten_2018_Kurzfassung.pdf; Stand: 29.05.2020

[2] BT-Drucksache 18/9528 vom 05.09.2016 - Deutscher Bundestag. Gesetzentwurf der Bundesregierung zur Weiterentwicklung der Versorgung und der Vergütung für psychiatrische und psychosomatische Leistungen (PsychVVG) 2018. http://dipbt.bundestag.de/dip21/btd/18/095/1809528.pdf; Stand: 29.05 .2020

[3] BT-Drucksache 18/10289 vom 09.11.2016 - Deutscher Bundestag. Beschlussempfehlung und Bericht des Ausschusses für Gesundheit (14. Ausschuss) 2018. http://dip21.bundestag.de/dip21/btd/18/102/1810289.pdf; Stand: 29.05.2020

[4] Grupp D. Politische Einordnung. In: Längle G, Holzke M, Gottlob M (Hrsg.) Psychisch Kranke zu Hause versorgen - Handbuch zur stationsäquivalenten Behandlung. 1. Aufl. Stuttgart: Kohlhammer; 2019: 49-51

[5] Holzke M, Gottlob M, Längle G. Stationsäquivalente Behandlung: Umsetzungserfahrungen der ersten 2 Jahre. Psychiatrische Pflege 2020; 5(3): 11-16

[6] Längle G, Holzke M, Gottlob M. Psychisch Kranke zu Hause versorgen. Handbuch zur stationsäquivalenten Behandlung. 1. Aufl. Stuttgart: Kohlhammer; 2019

[7] Sozialgesetzbuch (SGB) Fünftes Buch: Gesetzliche Krankenversicherung. http://www.gesetze-im-internet.de/sgb_5/ SGB_5.pdf; Stand 29.05.2020

[8] Deutsche Krankenhausgesellschaft, GKV Spitzenverband, Verband der Privaten Krankenversicherung. Vereinbarung zur stationsäquivalenten psychiatrischen Behandlung nach $\S 115$ d Abs.2 SGB V. 2017. https://www.dkgev.de/fileadmin/ default/Mediapool/2_Themen/2.3_Versorgung-Struktur/2.3.8._Psychiatrie-Pyschosomatik/2.3.8.2._Stationsaequivalente_psychiatrische_Behandlung/2017-08-01_Vereinbarung_StationsaequivalenteBehandlung.pdf; Stand: 29.05.2020

[9] Deutsche Krankenhausgesellschaft. Umsetzungshinweise der Deutschen Krankenhausgesellschaft zur Vereinbarung der Stationsäquivalenten Behandlung nach $\S 115 \mathrm{~d}$ Absatz 2 SGB V sowie ergänzende Informationen. 2017. https://www.dkgev.de/fileadmin/default/Mediapool/2_Themen/2.3_Versorgung-Struktur/2.3.8._Psychiatrie-Pyschosomatik/2.3.8.2._ Stationsaequivalente_psychiatrische_Behandlung/ Umsetzungshinweise_stationsaequivalente_Behandlung.pdf; Stand: 29.05 .2020

[10] Richtlinie des Gemeinsamen Bundesausschusses über die Verordnung von häuslicher Krankenpflege (Häusliche Krankenpflege-Richtlinie). https://www.g-ba.de/downloads/62-492-2133/HKP-RL_2020-03-20_iK-2020-05-08. pdf; Stand 29.05.2020

[11] Positionspapier der Verbände: Leistungsbeschreibung der stationsäquivalenten psychiatrischen Behandlung bei Erwachsenen. https://www.dgppn.de/_Resources/ Persistent/6a32b1531f474c898fc8ec49c7bf6614cb95ac15/2017-06-13_STN_DGPPN_ST\%C3\%84B_fin.pdf; Stand 29.05.2020

[12] Deutsches Institut für medizinische Dokumentation und Information. OPS Version 2020. https://www.dimdi.de/ static/de/klassifikationen/ops/kode-suche/opshtml2020/; Stand: 29.05 .2020

[13] Richtlinie des Gemeinsamen Bundesausschusses über die Ausstattung der stationären Einrichtungen der Psychiatrie und Psychosomatik mit dem für die Behandlung erforderlichen therapeutischen Personal gemäß §136a Absatz 2 Satz 1 des Fünften Buches Sozialgesetzbuch (SGB V) (Perso- 
nalausstattung Psychiatrie und Psychosomatik-Richtlinie/ PPP-RL). https://www.g-ba.de/downloads/62-492-2120/ PPP-RL_2020-03-27_iK_2020-03-27_AT-08-04-2020-B4.pdf; Stand 29.05.2020

[14] Hölling H, Schlack R, Petermann F et al. Psychische Auffälligkeiten und psychosoziale Beeinträchtigungen bei Kindern und Jugendlichen im Alter von 3 bis 17 Jahren in Deutschland - Prävalenz und zeitliche Trends zu 2 Erhebungszeitpunkten (2003-2006 und 2009-2012). Bundesgesundheitsblatt 2014; 57: 807-819

[15] Boege I, Schepker R, Grupp D et al. Kinder-und jugendpsychiatrische stationsäquivalente Behandlung (StäB): Therapieoption - für alle oder für wenige?. Zeitschrift für Kinder- und Jugendpsychiatrie und Psychotherapie 2020; 1-10. doi: 10.1024/1422-4917/a000711

[16] Boege I. Stationsäquivalente Behandlung in der Kinder- und Jugendpsychiatrie. In: Längle G, Holzke M, Gottlob M (Hrsg.) Psychisch Kranke zu Hause versorgen - Handbuch zur stationsäquivalenten Behandlung. 1. Aufl. Stuttgart: Kohlhammer; 2019: 57-62

[17] Fegert JM, Kölch M, Krüger U. Versorgung psychisch kranker Kinder und Jugendlicher in Deutschland - Bestandsaufnahme und Bedarfsanalyse (2017). https://www. apk-ev.de/fileadmin/downloads/Abschlussbericht_Versorgung_psychisch_kranke_Kinder_u_Jugendliche.pdf; Stand: 29.05.2020

[18] Boege I, Schepker R, Fegert JM. Vom Hometreatment zur stationsäquivalenten Behandlung (StäB). Zeitschrift für Kinder-und Jugendpsychiatrie und Psychotherapie 2020; 1-12. DOI: $10.1024 / 1422-4917 / \mathrm{a} 000710$

[19] Mattejat F, Hirt BR, Wilken J et al. Efficacy of inpatient and home treatment in psychiatrically disturbed children and adolescents. Follow-up assessment of the results of a controlled treatment study. Eur Child Adolesc Psychiatry 2001; 10(1): S71. doi: 10.1007/s007870170008

[20] Schmidt MH, Lay B, Göpel C et al. Home treatment for children and adolescents with psychiatric disorders. Eur Child Adolesc Psychiatry 2006; 15(5): 265-276. doi: 10.1007| s00787-006-0531-x

[21] Gesetz zur wirtschaftlichen Sicherung der Krankenhäuser und zur Regelung der Krankenhauspflegesätze (Krankenhausfinanzierungsgesetz - KHG). https://www.gesetze-im-internet.de/khg/KHG.pdf; Stand: 29.05.2020

[22] Deutsche Krankenhausgesellschaft, GKV Spitzenverband, Verband der Privaten Krankenversicherung. Vereinbarung zum pauschalierenden Entgeltsystem für psychiatrische und psychosomatische Einrichtungen für das Jahr 2020 (Vereinbarung über die pauschalierenden Entgelte für die Psychiatrie und Psychosomatik 2020 - PEPPV 2020) https:// www.gkv-spitzenverband.de/media/dokumente/krankenversicherung_1/krankenhaeuser/psychiatrie/pepp/PEPPV_2020. pdf; Stand: 29.05.2020

Bibliografie

DOI https://doi.org/10.1055/a-1199-7577 Nervenheilkunde 2020; 39: 704-710 (C) 2020. Thieme. All rights reserved. Georg Thieme Verlag KG, Rüdigerstraße 14, 70469 Stuttgart, Germany ISSN 0722-1541 


\section{Punkte sammeln auf CM/F.thieme.de}

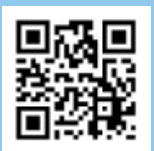

Diese Fortbildungseinheit ist bis zu 12 Monate online für die Teilnahme verfügbar.

Den genauen Einsendeschluss finden Sie beim Modul auf https://cme.thieme.de/CXF9AK1.

Sollten Sie Fragen zur Online-Teilnahme haben, finden Sie unter https://cme.thieme.de/hilfe

eine ausführliche Anleitung. Wir wünschen viel Erfolg beim Beantworten

der Fragen!

Unter https://eref.thieme.de/CXF9AK1 oder über den QR-Code kommen Sie

direkt zum Artikel.

VNR 2760512020158722336

\section{Frage 1}

Welche Aussage trifft nicht zu?

A StäB ist eine Form der aufsuchenden Akutbehandlung ausschließlich für Patienten mit einer stationär behandlungsbedürftigen Psychoseerkrankung.

B StäB ist eine Form der aufsuchenden Akutbehandlung für Patienten mit einer stationär behandlungsbedürftigen Suchterkrankung.

C StäB ist eine Form der aufsuchenden Akutbehandlung für Patienten mit einer stationär behandlungsbedürftigen psychiatrischen Erkrankung.

D StäB ist eine Form der aufsuchenden Akutbehandlung für Patienten mit einer stationär behandlungsbedürftigen Angststörung.

E StäB ist eine Form der aufsuchenden Akutbehandlung für Patienten mit einer stationär behandlungsbedürftigen Demenzerkrankung.

\section{Frage 2}

Die stationsäquivalente Behandlung entspricht hinsichtlich der Inhalte, Flexibilität und Komplexität der Behandlung einer ...
A ambulanten Behandlung.
$B$ vorstationären Behandlung.
C nachstationären Behandlung.
D tagesklinischen Behandlung.
E vollstationären Behandlung.

\section{Frage 3}

Die Bundesrahmenvereinbarung regelt das Mindestmaß der Behandlungsfrequenz und -intensität der StäB. Mindestens vorgehalten werden muss/müssen ...

A ein telefonischer Kontakt in 2 Tagen.

B ein persönlicher Kontakt pro Woche.

C ein persönlicher ärztlicher Kontakt pro Tag.

D ein direkter persönlicher Kontakt durch ein Mitglied des Behandlungsteams pro Tag.

E 3 persönliche Kontakte pro Woche.

\section{Frage 4}

Bezüglich der Einbindung außerklinischer Leistungserbringer schreibt die Rahmenvereinbarung Folgendes vor:

A Mindestens $50 \%$ der Behandlungstätigkeiten sollen an auBerklinische Leistungserbringer delegiert werden.

B Außerklinische Leistungserbringer dürfen nicht an der stationsäquivalenten Behandlung beteiligt werden.

C Maximal die Hälfte der Behandlungstätigkeiten dürfen an auBerklinische Leistungserbringer delegiert werden.

D Außerklinischen Leistungserbringern, welche mindestens $50 \%$ der Behandlungstätigkeiten übernehmen, wird die Gesamtverantwortung für den Fall übertragen.

E Als außerklinische Leistungserbringer dürfen ausschließlich betreute Wohneinrichtungen oder der sozialpsychiatrische Dienst an der stationsäquivalenten Behandlung beteiligt werden.

\section{Frage 5}

Welche Einrichtungen sind grundsätzlich berechtigt StäB anzubieten?

A Kliniken für Psychiatrie und Kliniken für Kinder- und Jugendpsychiatrie

B Niedergelassene Psychotherapeuten

C Fachpsychiatrische Gemeinschaftspraxen

D Psychosomatische Krankenhäuser

E Kliniken für forensische Psychiatrie

\section{Frage 6}

Welche Kriterien sind bei einer StäB nicht zwingend erforderlich?

A Permanente Präsenz eines Angehörigen oder Mitbewohners.

$B$ Ausschluss einer Kindeswohlgefährdung.

C Einverständnis der volljährigen im selben Haushalt lebenden Personen.

D Eignung des häuslichen Umfeldes.

E Wöchentliche multiprofessionelle Fallbesprechungen. 


\section{Punkte sammeln auf CME.thieme.de}

Fortsetzung ...

\section{Frage 7}

Im Spektrum psychiatrischer Behandlungsangebote stellt die StäB am ehesten eine ...

A intensive und wohnortnahe Behandlung dar.

$B$ wenig intensive und wohnortnahe Behandlung dar.

C wenig intensive und wohnortferne Behandlung dar.

D intensive und wohnortferne Behandlung dar.

E auf ausschließlich ausgewählte Diagnosegruppen spezialisierte, wohnortferne Behandlung dar.

\section{Frage 8}

Welche Aussage trifft nicht zu?

A Neu auftretende Begleiterkrankungen liegen in der Zuständigkeit des StäB-Teams.

B Häusliche Krankenpflege ist Teil der StäB-Leistung und muss aus dem StäB-Team heraus erbracht werden.

C Bei neu auftretenden Begleiterkrankungen muss eine stationäre Aufnahme erfolgen.

D Leistungen des ambulant betreuten Wohnens fallen nicht in die Zuständigkeit des StäB-Teams.

E Leistungen des Sozialpsychiatrischen Dienstes fallen nicht in die Zuständigkeit des StäB-Teams.

\section{Frage 9}

Die Finanzierung für die StäB ...

A ist bundesweit einheitlich geregelt.

$B$ ist länderspezifisch einheitlich geregelt.

$C$ ist bislang für kein Krankenhaus geregelt.

D ist immer identisch mit dem jeweiligen stationären Pflegesatz.

E liegt in der Verantwortung der Pflegesatzverhandlungen vor Ort.

\section{Frage 10}

Welche Aussage für die StäB-Behandlung während der Corona-Pandemie trifft zu?

A Während der Corona-Pandemie sind aufsuchende Behandlungen verboten.

B Eine Reduktion der Behandlungsfrequenz von täglichen Kontakten auf wöchentliche Kontakte wurde während der Corona-Pandemie in StäB ermöglicht.

C Für StäB gelten dieselben Hygiene- und Abstandsregelungen wie bei einer stationären Behandlung.

D Aus arbeitsschutztechnischer Sicht ist die StäB während der Corona-Pandemie unzulässig.

E Auf Schutzmaßnahmen kann in der aufsuchenden Behandlungssituation verzichtet werden. 